\title{
ESTRATEGIAS FAMILIARES DE VIDA Y VIOLENCIA: HISTORIAS DE VIDA EN LAS COMUNIDADES LA YABITA Y CERRO VERDE DE HOLGUÍN
}

\section{FAMILY LIFE STRATEGIES AND VIOLENCE: STORIES OF LIFE IN THE COMMUNITIES THE YABITA AND CERRO VERDE OF HOLGUIN}

\author{
Elsie Alejandrina Pérez Serrano ${ }^{1}$
}

\section{RESUMEN}

Las estrategias que las familias utilizan para vivir en condiciones de pobreza se han estudiado insuficientemente en Cuba y no se visibiliza las formas de violencia que les acompañan. Como parte del proceso de restratificación social que se origina en el país a partir de la década de 90 , se acentúa el proceso migratorio del campo con el consiguiente asentamiento de familias en los márgenes de las ciudades, donde la pobreza se reproduce de una generación a otra. En el trabajo se realiza un acercamiento a las estrategias familiares de vida en estas personas a través de dos historias de vida en las comunidades: La Yabita y Cerro Verde del municipio de Holguín. En la construcción de las historias de vida de una mujer y un hombre, se evidencia el estado de sobrevivencia en que transcurre la existencia de estas familias y como la violencia forma parte de su cotidianidad en lo privado y lo público, lo cual no favorece el tránsito de la cultura de la pobreza a la de la transformación.

Palabras claves: Pobreza. Violencia. Estrategias familiares de vida. Periferia urbana y cultura de la pobreza.

\begin{abstract}
The strategies that families used to live in poverty conditions have been insufficiently studied in Cuba and the forms of violence that accompany them are not made visible. As part of the process of social restratification that arise in the country since the ' $90 \mathrm{~s}$, the migration process from the field increases, with the consequent settlement of families on the suburbs, where poverty is reproduced from one generation to another. In this paper an approach to the family life strategies of these people through two stories of life in communities - La Yabita and Cerro Verde, in the city of Holguín - is carried out. In the construction of the life stories of a woman and a man, it is possible to notice the state of survival that accompanies the existence of these families and how the violence is part of their private and public daily lives, which does not favor the transition from the culture of poverty to the culture of transformation.
\end{abstract}

Keywords: Poverty. Violence. Family life strategies. Suburbs and culture of poverty.

1 Doctora en Ciencias Pedagógicas, Profesora Titular de la Universidad de Holguín. Ha sido Profesora/investigadora en Centro de Estudios de Ciencias de la Educación y del Centro de Estudios sobre Cultura e Identidad, actualmente es miembro del Grupo de Desarrollo Local del Centro de Estudios en Gestión Empresarial. Bolsista CAPES/Brasil. E-mail: eperez@fh.uho.edu.cu y elsieperezserrano@gmail.com 


\section{Introducción}

El Siglo XXI está marcado por procesos de globalización que median el avance de las naciones. Como respuesta a los efectos negativos de este proceso surgen múltiples concepciones del desarrollo, entre las cuales el desarrollo local se enfila hacia temas como la pobreza, la marginalidad, la violencia, las migraciones del campo a la ciudad y el papel de la mujer en cada uno de los procesos.

La familia es central en el progreso de los seres sociales en todos los momentos del ciclo familiar y a su interior se modela la apreciación de la realidad a través de patrones, representaciones y percepciones afirmados de una generación a otra. Con ello la familia legitima sus concepciones y posición en la estructura social, en lo cual la reproducción social es un proceso básico.

En el tema de reproducción social es ineludible la obra del sociólogo francés P. Bourdieu y su teoría de las prácticas sociales, desarrollada a través de los conceptos de habitus, campo y capital en sus diferentes variantes. La perspectiva teórica de P. Bourdieu permite estudiar desde conceptos claves las estrategias familiares de vida, como la relación del espacio social con la construcción de estas estrategias. Las familias en situaciones de crisis urden múltiples recursos para solucionar sus necesidades, sin estar conscientes de que organizan estrategias para salvarse de la crisis.

A nivel internacional la problemática de las estrategias familiares se ha estudiado, sobre todo a partir de las décadas de los años 70 y 80 en países latinoamericanos como Argentina, Brasil, Chile y México. Entre los autores que más aportan desde la teoría y la metodología se encuentran S. Hintze (s/f), S. Torrado (1985), M. Molina (2006), A. Gutiérrez (2007), C. Arteaga (2007) y el sociólogo francés P. Bourdieu (1994).

La emergencia de este tema en países latinoamericanos no es casual, surge como respuesta al deterioro del estado de bienestar y la implementación de las políticas de ajuste estructural. Bajo estas circunstancias las familias de bajos ingresos, construyen procederes para salvaguardarse de la pobreza y tratar de satisfacer sus necesidades. Estas estrategias son diferentes en hombres y mujeres, generalmente asociadas a los roles que les son asignados tradicionalmente.

Los estudios acerca de estas estrategias en Cuba son incipientes. En la bibliografía consultada se encuentran resultados desde la psicología y la sociología, donde destacan M. Díaz (2005) y M. Zabala (2010). Un estudio valioso es el de M. Arias (2011), desarrollado como profesor investigador por CAPES, en Brasil.

La crisis vivida por la familia cubana en la década de los años 90 ha dejado huellas en su desarrollo actual y futuro. La principal evidencia de esta situación radica en que a pesar de haber transcurrido más de una década, todavía el país no logra recuperarse $\mathrm{y}$ algunos de los problemas aún persisten. Este constituye uno de los principales motivos del estudio en comunidades de mayor vulnerabilidad social, tanto rurales como de la periferia urbana, realizado por investigadores del Grupo de Desarrollo Local, la Cátedra de Antropología y el Núcleo de Estudios de Género de la Universidad de Holguín en Cuba.

La investigación se realiza en las comunidades de Camarones, Cerro Verde y La Yabita, donde se estudia la situación laboral en que viven sus habitantes, principalmente las mujeres y la proyección de vías para favorecer mejoras económicas. El objetivo es caracterizar las estrategias familiares de vida en el proceso de reproducción social con enfoque de género en las comunidades referidas y visibilizando otros procesos asociados a poblaciones emergentes como la violencia. Para ello la investigación se asume desde el método dialéctico-materialista, a través de una metodología cualitativa en la cual se valoran y triangulan diversos métodos y técnicas de investigación teniendo como núcleo las historias de vida.

Distinguir el estatus rural, urbano o rururbano de las comunidades como en las que se realiza el estudio es objeto de discusión de los teóricos actualmente. Los elementos que con mayor frecuencia se enuncian para caracterizar lo urbano son: el tamaño y la densidad, el aspecto del núcleo, la actividad no agrícola y el modo de vida, así como ciertas características sociales, tales como la heterogeneidad, la "cultura urbana" y el grado de interacción social. ${ }^{2}$

2 Capel, H. (1975). La definición de lo urbano. En: Estudios geográficos n. $138-139$, p. 265 


\section{Estrategia metodológica utilizada para la construcción de las historias de vida}

Para la utilización de las historias de vida se acoge el criterio de G. Rodríguez, et al (1995) quien las considera parte del método biográfico junto a los documentos personales, los relatos de vida y los biogramas. Se comparte su opinión de que este método ayuda a conocer las experiencias vitales del individuo investigado y la interpretación subjetiva que el mismo hace de estas experiencias, así como las etapas por las que transita el método. El autor tomando como referencia a Pujadas (1992) establece una distinción con el relato de vida el cual es: ... la historia de una vida tal y como la persona que la ha vivido la cuenta; y la historia de vida, que se refiere al estudio de caso de una persona dada, comprendiendo no solo su relato de vida... ${ }^{3}$

También, se toman como presupuesto teóricometodológico las opiniones de H. Saltalamacchia (1992) sobre varios aspectos, entre ellos que la muestra puede seleccionarse sobre la base de conceptos ordenadores que aprehenden las distinciones dentro de la sociedad, los cuales determinan la existencia de los agentes sociales y su conducta individual en las clases como la edad, el sexo y la región a la que pertenecen. Se toma como guía sus ideas acerca de la reconstrucción del sentido por la percepción activa del sujeto a partir de los valores, saberes y certezas que comparte con sus contemporáneos o solo con algunos de ellos. Igualmente las bases que expresa sobre la postura del entrevistador para evitar la ocurrencia de problemas en la realización de la entrevista, como espacio de producción de una relación social.

Un requisito en todo estudio es el rigor de la investigación y teniendo en cuenta que se utiliza una metodología cualitativa, se adoptan los criterios de confiabilidad propuestos por E. Guba según referencia de M. Pla (1992). Estos criterios son credibilidad, transferibilidad, dependencia o consistencia y confirmabilidad, y se asumen los procedimientos expuestos por el autor para cada uno de ellos. De esta manera, siempre participan

3 Rodríguez Gómez, G.; Gil Flores, J. y García Jiménez, E. (1995). Metodología de la investigación cualitativa. La Habana: Félix Varela, p.58. dos investigadores y para la caracterización de las comunidades y los sujetos seleccionados se utilizan otras técnicas como la observación no participante y la revisión de documentos. También, entrevistas a los actores sociales entre los cuales destacan el trabajador social, la presidenta de la organización de las mujeres (Federación de Mujeres Cubanas) y el o la presidente de la organización barrial (Comité de Defensa de la Revolución). La metodología utilizada se estructura en tres pasos que se explican brevemente.

Primer paso, selección de la muestra que como aspecto central en la construcción de las historias de vida, solo es encontrada al finalizar la investigación $\mathrm{y}$, a la vez, se convierte en uno de sus resultados. Para elegir el tipo de personas que se pretende en las comunidades de estudio se formulan los criterios de inclusión que se relacionan:

- Residencia en la comunidad de al menos dos generaciones de la familia.

- Representación de ambos sexos.

- Diferentes tipos de familia.

- Utilización de diversas estrategias familiares de vida.

- Múltiples formas de violencia, visible y no visible, en diferentes dimensiones de su existencia.

Como criterio de exclusión se enuncian dos esencialmente, la no disposición de participar en el estudio o la movilidad de residencia hacia otra comunidad ya sea de forma temporal o permanente. Por estos criterios la muestra final difiere de la seleccionada inicialmente.

Segundo paso, preparación de la entrevista que requiere de un proceso de familiarización con el contexto por lo cual se planifican, al menos, dos visitas previas para el conocimiento del escenario y los entrevistados, negociar el acceso y concertar la primera entrevista. Como momento de máxima responsabilidad del investigador se tienen en cuenta los aspectos siguientes:

Producción de datos pertinentes mediante fuentes secundarias: los datos recogidos mediante otras técnicas proporcionan un material de importancia que complementa la investigación, entre estos se encuentran: la cronología del período 
investigado, el historial del trabajador social y la clasificación de las principales líneas del conflicto que influyen en la conducta de los entrevistados. Para ello se atiende a los elementos que se re relacionan:

- Diseñar preguntas y temas problemáticos para si fuese necesario recurrir a ellas.

- Disponer de explicaciones de los hechos más difundidos puespueden organizar la interpretación del entrevistado y suplantar su memoria.

- Discernir las experiencias destacadas de la vida de los entrevistados y la interpretación que hace de ellas.

A. Guía de la entrevista: de tipo semiestructurada por lo cual no se lleva ninguna guía escrita sino temas generales, lo cual requiere de una minuciosa preparación del entrevistador, siendo esenciales los aspectos siguientes:

- Preparar la vía para establecer la empatía y ganar la confianza de los entrevistados.

- Planificar la manera de realizar la consulta para no llamar demasiado la atención de la persona entrevistada.

- Planear un lenguaje sencillo con una terminología asequible al contexto de vida de estas personas.

B. Determinación del lugar en que se llevará a cabo la entrevista: para Tina y Antonio, nombre ficticio atribuido a los entrevistados al igual que la nominación del resto de los familiares mencionados se elige su propia casa, lo cual se justifica por los aspectos siguientes:

- El lugar permita su desarrollo de forma espontánea y tranquila.

- El contexto es parte de la situación y de la historia de estas personas.

- La preparación de una situación de familiaridad que les permita expresarse libremente.

Para Tina se selecciona el patio frontal de la vivienda ubicada en un lugar de poco tránsito de vecinos y medios de transporte, además resguardado por plantas, y en Antonio se escoge un corredor al fondo para evitar interrupciones por el paso de los transeúntes.

Tercer paso, desarrollo de las entrevistas para lo cual se coincide con B. Sarabia (1985), F. Mallimaci y V. Giménez (2006) y H. Saltalamacchia (1992) de que se deben realizan un mínimo de tres. En estas se lleva un registro de los elementos que se asocian con los cambios en el discurso y el lenguaje extraverbal, igualmente se consignan los gestos y mímica, los silencios, la mirada, la entonación, la expresión corporal y sonidos como toser, carraspear y reír, entre otros. Cada una de las entrevistas tiene sus objetivos específicos y una etapa posterior de análisis con fines diferentes, todo lo cual se precisa brevemente:

En la primera entrevista, la participación del entrevistador es mínima para no interferir en el curso de la memoria del sujeto y las recreaciones que surjan espontáneamente. Durante la remembranza del entrevistado solo se hacen interrupciones para encausar su discurso si se aleja demasiado y para centrar su atención en períodos poco abordados. El orden de acciones a seguir es el siguiente:

- Se informan los orígenes, objetivos y métodos de la investigación, nuevamente se le solicita su colaboración y la firma del consentimiento informado.

- Se solicita que recuerde -lo más posible en forma cronológica- todo lo referido a su vida $y$, en la medida que el discurso transcurre, se realizan preguntas para destacar acontecimientos relacionados con el tema de la investigación y solicitarle anécdotas.

- Al finalizar, se le agradece su participación en la investigación y se concilia la segunda entrevista.

A continuación el investigador ejecuta un análisis preliminar del texto resultante con el objetivo de preparar la segunda entrevista. Este análisis consta de dos etapas: en la primera se detalla el texto de la entrevista a través de varias lecturas con sus anotaciones y se delimitan un conjunto de elementos que pueden esclarecer el trabajo del investigador, entre los que se encuentran: 
- Descubrir probables incongruencias en la información del testimoniante.

- Detectar silencios significativos sobre acontecimientos que supuestamente el entrevistado debe conocer y recordar, pero no ha narrado.

- Reconocer cuando el investigador haya cometido una racionalización ex post facto.

- Identificar ideas que han sido insuficientemente abordadas pero son de importancia para la investigación.

En la segunda etapa se debe comparar el texto de la entrevista con el texto preparado anteriormente a partir de la información proporcionada por otras fuentes. El resultado de ambas fases crea las condiciones para elaborar la guía de la entrevista siguiente.

En la segunda entrevista, el entrevistador ocupa un rol más activo para precisar los resultados de la primera entrevista. El propósito de esta es cumplir lo más ajustadamente posible con la guía que ha sido preparada como fruto del análisis de la primera entrevista, para lo cual se sugiere:

- Elaboración conjunta de los problemas que resultan importantes.

- Énfasis en contenidos significativos de las acciones narradas para descubrir la influencia del conocimiento posterior en la narración.

- Elucidación con el entrevistado de interpretaciones alternativas al testimonio.

Nuevamente, se realiza otro análisis del texto ya enriquecido y que es una versión más acabada de la historia de vida. Igualmente, se puede elaborar preguntas ante pasajes con los cuales se tengan dudas.

En la tercera entrevista, se devuelve a los entrevistados una primera versión del informe con los principales elementos de la construcción, en este caso a cada uno por separado. El texto debe reflejar las pautas expresivas características, las construcciones gramaticales y el estilo personal del entrevistado, para lo cual se incluyen citas textuales. Este momento de la construcción de la historia de vida permite:

- Someter la historia reconstruida a la crítica de quienes la vivieron.
- Incitar a nuevas elaboraciones ante la narración global.

- Ofrecer elementos para autorizar su publicación o la inclusión de sus nombres reales.

De los resultados obtenidos en las entrevistas, la confrontación de la información obtenida por los restantes métodos, instrumentos y técnicas así como todas las explicaciones logradas al final de la investigación, se elabora la caracterización de las comunidades referidas y una síntesis de las historias de vida seleccionadas para este trabajo. Una versión resumida se muestra en el presente trabajo.

\section{Caracterización de la comunidad La Yabita}

La Yabita es una comunidad de inmigrantes pobres que se encuentra ubicada en la zona norte del municipio Holguín. Pertenece al Consejo Popular Alcides Pino, el cual cuenta con una extensión de 4,2 Km2 y una población total de 33. 666 habitantes. De esta población, 16. 849 son mujeres y 16. 817 son hombres, constituyendo el Consejo Popular más poblado de la ciudad de Holguín. La Yabita es un barrio ubicado en la periferia urbana que tiene como características la inestabilidad económica la cual genera pobreza, la violencia desencadenada por la ingestión de bebidas alcohólicas, la presencia de prejuicios hacia sus habitantes y como consecuencia representaciones colectivas que afectan la imagen de sus pobladores, dejándolos en un estado de inestabilidad.

El Consejo de Administración Municipal considera aestacomunidad comounadelas de mayores problemas sociales, debido a múltiples factores económicos, sociales, culturales, educacionales y de salud. En ella no existen empresas o industrias que generen fuente de empleos, no cuenta con escuelas, ni tiendas de suministros de víveres, hortalizas u otro tipo de alimentos. Solo hay un centro agente para la comunicación telefónica que lo atiende la esposa del joyero, la figura de mayor poder económico en el entorno social.

La comunidad no posee un trazado urbano ni alcantarillado, ni acueducto $\mathrm{u}$ otros servicios que brinden calidad de vida a sus pobladores. Presenta 
dos núcleos delimitados, uno al norte con mejores casas y habitantes con empleos como una enfermera, una maestra, trabajadores de servicios y comunales, entre otros; y uno al sur de mayor pobreza cuyos moradores carecen de entrada económica estable, más casas dispersas y otras pequeñas aglomeraciones.

Las personas están mayormente desempleadas y desvinculadas del estudio, en el caso de las mujeres es superior al $90 \%$, por lo cual habitan en las condiciones de vida mínimas. Es común la convivencia de familias múltiples con varios niños y niñas las cuales disponen como único ingreso estable una pensión de la asistencia social.

Los habitantes no solo tienen carencias materiales, sino educacionales, debido a que prevalece el noveno grado en la población. La violencia en el hogar es común, pues las mujeres no asumen que el maltrato o el habla en mala forma constituyen tipos de violencia verbal o psicológica y solo reconocen las manifestaciones de violencia física. Durante entrevistas desarrolladas en la comunidad, las mujeres expresaron la conformidad de algunas de ellas al ser violentadas y la posición de que: las demás personas no tienen que meterse en mis asuntos.

\section{Síntesis de la historia de Tina}

Tina nació en 1957 en el actual Consejo Popular Alcides Pino de Holguín. Su madre fue ama de casa, su padre hacía carbón para vender y dormían en un saco en el suelo. Tuvo doce hermanos, cuatro hembras y ocho varones quienes la obligaban a que les lavara la ropa y si no le pegaban. Esta situación generaba discusiones familiares, debido a que la madre decía: no lave' na'. El padre le llegó a pegar a uno de los hijos para evitar que golpearan a Tina. Este ambiente se repetía hasta poco antes de casarse con el padre de sus hijos.

A los 15 años conoció a Trujillo con quien se casó enseguida y tuvieron cinco hijos. Dos años después se mudaron para La Yabita. La casa es de piso de tierra, paredes de madera, tiene un patio grande cercado con mata de cardona, sin portería ni jardín y con algunas plantas de plátano, ciruela y un árbol de mango. El acceso al servicio eléctrico es mediante una tendedera ilegal y solo tienen un televisor pequeño en blanco y negro que cuando salen todos de la casa lo dejan al cuidado de la vecina y ella hace lo mismo. La ropa que la familia usa se la regalan.

Todos los hijos de Tina están casados y viven independientes, excepto la más joven, Ana, de 17 años. Los hijos de Tina no tienen trabajo estable pues venden en las calles y ahora se dedican a la minería ilegal. Solo Oscar quien antecede a Ana, terminó el bachillerato es el único que les visita y ayuda, pues los demás: imagínese la vida está dura y cada cual se ocupa de lo suyo.

Cuando Ana cumplió los 15 años, unas personas allegadas le regalaron una muda de ropa y su padrino pagó las fotos y ninguno de sus hermanos fue a verla. Ella está esperando por un hombre: que se lleva bacán conmigo me consiga de secretaria o recepcionista para poder entrar un dinerito. Ana abandonó los estudios porque un día el padre la avergonzó delante de los compañeros de escuela, diciendo palabras soeces en alta voz. Después de esto Ana no quiso volver a la escuela porque los demás se iban a reír de ella.

La casa es de madera, yagua y techo de zinc sin letrina pues solo: cogen pa'1 monte. Está constituida por una primera habitación con doble función, cuarto del matrimonio y sala de la casa. Le continúan dos cuartos, uno pequeño de Ana y otro donde guardan las materias primas acumuladas hasta que se llevan a vender y un pasillo que conduce a la cocina ubicada al final con un fogón de leña, dos sillas desvencijadas y una mesa ancha y baja en la que coloca las ollas tiznadas pues: $p a^{\prime}$ que las voy a fregar si se ensucian otra ve'.

Tina es ama de casa y recoge materias primas (plástico, laticas, trozos de tela y cartón) que vende en los establecimientos para dichos cambios y la tela a trabajadores cuentapropistas para la construcción de muebles. El esposo trabaja en una cochiquera particular durante las 24 horas del día excepto el domingo, él lava los corrales y alimenta a los animales de día; de noche hace guardia cuidando la cría, no recibe alimentos, duerme cerca de la porqueriza y le pagan 500 pesos al mes pero no lo deja o pide mejoras porque: si no morimos de hambre. 
Tina se ocupa de los quehaceres del hogar, ir a la bodega que se encuentra a casi un kilómetro, ayuda en los gastos de la casa, diariamente lleva la comida a su esposo y la mayoría de las noches lo acompaña en las guardias.

En la casa de Tina pocas veces desayunan y consiste en un café claro, en contadas ocasiones almuerzan y cuando lo hacen es una yuca o calabaza hervida. Los alimentos que poseen lo reservan para la comida de la tarde. El rostro de Tina refleja desamparo y ante muchas preguntas sonríe con una expresión primitiva.

\section{Caracterización de la comunidad Cerro Verde}

La comunidad Cerro Verde se encuentra ubicada en la zona norte del municipio Holguín. Pertenece al Consejo Popular Aguas Clara que colinda con el de Alcides Pino. Cuenta con una extensión de $82 \mathrm{~km} 2$ y una población total de 7 . 015 habitantes, de ellos 2. 656 son mujeres y 3 . 245 son hombres y 1. 114 niños. Tampoco, posee un trazado urbano, ni alcantarillado, ni acueducto, ni espacios para la recreación y el esparcimiento. Constituye un conglomerado de casas más o menos ordenadas y agrupadas alrededor de la bodega, un pequeño mercado agropecuario, un consultorio del médico de la familia, un merendero y un taller de reparaciones.

La principal vía de comunicación es la carretera a Gibara que enlaza el Consejo Popular con el centro de la ciudad de Holguín atravesando el de Alcides Pino y sirve de eje a los dos Consejos. Además, existen calles tortuosas en su mayoría de tierra o cuyo asfalto se ha deteriorado por el tiempo. La comunidad Cerro Verde posee un centro agente, la escuela primaria Ricardo Martínez Alvares, un anexo de la escuela secundaria y una fábrica de bloques. Ante la escasez de fuente de empleo la movilidad hacia el centro de la ciudad de Holguín es constante $\mathrm{y}$ : los vecinos ni se conocen ni se saludan.

El nivel escolar en general es de noveno grado. Entre los principales problemas se encuentran el alto índice de familias disfuncionales, los adultos sin vínculo laboral, los exreclusos, las deudas con el banco, la poca atención a los adultos mayores y la falta de recreación sana. Uno de las dificultades crecientes es el alcoholismo que imposibilita el vínculo laboral y contribuye al incremento de las indisciplinas sociales pues en las bebederas se pasa de la violencia verbal a la física.

Los padres cohabitan sin contraer matrimonios formales y el divorcio es frecuente, quedando los hijos a la guarda de las madres quienes en su mayoría dependen de la pensión a los hijos, de trabajos ilegales o de uniones consensuales transitorias. Las relaciones de los padres divorciados con sus hijos no son buenas, porque estos hijos poseen una imagen negativa de sus padres, fomentada por la madre, la familia materna y a veces por el tutor.

La situación descrita crea las bases para el mantenimiento de la pobreza, la cual encuentra otras fuentes de reproducción en el bajo nivel cultural de los pobladores de la comunidad, esencialmente los padres como figura de sostén económico. En este caso, la mayoría de los padres solo ha culminado la Secundaria Básica, por lo que existe una incorporación temprana al trabajo mal remunerado y encuentra en las prácticas económicas ilegales la vía para suplir las necesidades propias y de la familia.

\section{Síntesis de la historia de Antonio}

Antonio nació en 1965 en el Consejo Popular Aguas Claras del municipio de Holguín, actualmente vive en el barrio Cerro Verde. Es el menor de tres hermanos y siempre fue el preferido por la madre que lo sobreprotege en todo, lo cual originó conflictos con el padre.

La casa es de mampostería, posee tres cuartos y demás dependencias, con letrina sanitaria en buen estado. Sus hermanos tienen casa propia y él vive solo con su madre pues el padre, quien era custodio en la mina, cerrada actualmente, falleció hace 10 años a consecuencia de un infarto.

Antonio culminó el noveno grado, nunca ha trabajado legalmente y se dedica a buscar oro en un yacimiento que está en Aguas Claras y es propiedad de la Empresa Geominera de Oriente. A pesar de conocer lo ilegal de dicho trabajo y tener varias advertencias policiales continúa haciéndolo, corriendo el riesgo de ser apresado o simplemente 
deja de ir un tiempo cuando la policía frecuenta el lugar: para retornar cuando la poli nos deja en paz.

Hace cuatro años mantiene relaciones con Maritza, seis años mayor que él quien emigró de Managuaco, barrio de Gibara con su hija. Ambos toman alcohol y dice una vecina que están juntos hasta que: forman la vaquera y se va cada uno para su casa. Estas discusiones han terminado en el hospital con lesiones graves para ella. Cuando tienen dinero compran de todo y después lo venden a menor precio pues dice Antonio que: para comelatas, tomar y fiestear pues total uno se va a morir. En una discusión lanzaron el TV pantalla plana para la carretera.

La labor de minería ilegal que Antonio despliega la realiza con equipos artesanales utilizando picas y palas y con el consiguiente daño al ecosistema. Él pasa casi ocho horas dentro del agua y separa las partículas de oro con mercurio que se absorbe por la piel y del cual emanan gases tóxicos. Para obtener más productos muchas veces Maritza le acompaña ya que: hay que luchar juntos para comer y vivir mejor.

La madre de Antonio dice: mi hijo es bueno, esa mujer tiene la culpa de todo y de la mala bronquitis que le da. La madre y Maritza discuten con frecuencia y ha habido que separarlas cuando el pleito sube de tono. Luego ambas lloran y se amigan: por el amor a Antonio, como dice Maritza. Antonio no les hace caso, refunfuña y dice que: estoy cansa'o de esta vida y un día me cogen monta'o, me voy y no vuelvo más.

\section{Valoración general de las comunidades y las familias investigadas}

A partir de los presupuestos teóricos de la obra de P. Bourdieu se aprecia correspondencia entre la manera de concebir la realidad y el medio en que viven los entrevistados y la comunidad en su conjunto. El medio en que se desarrollan la mayor parte de los habitantes es semejante al que producen y reproducen en la vida adulta.

La realidad social de estas comunidades es un ciclo cerrado, en el cual las acciones desempeñadas por los agentes se encuentran, entre la educación por familiarización y el medio que seleccionan para desarrollar sus vidas. Estas personas tienen esquemas similares de acción, pues se han formado bajo condiciones objetivas análogas, como: vulnerabilidad económica y social, y falta de calificación profesional lo cual genera bajos ingresos y baja autoestima, perdiendo confianza en sus proyectos y estrategias, muchos viven el día a día sin concebir proyecto alguno.

La pobreza constituye un elemento de la realidad cotidiana y las condicionantes expuestas ayudan a la permanencia prolongada de la familia en un mismo nivel del estrato social, ocupado de generación a generación. No hay evolución de las generaciones más jóvenes respecto a las adultas, con elementos que enriquezcan las especies de capital económico, social, cultural o simbólico. De esta forma, solo alcanzan asemejarse más a la generación precedente.

El habitus programa y sugiere lo que los agentes sociales perciben como necesario y en función de ello construyen estrategias para lograr sus objetivos. Estas estrategias referencian el comportamiento de los agentes sociales para la constitución y mantenimiento de las unidades familiares a través de las cuales se realiza la reproducción biológica y las prácticas, económicas y no económicas, indispensables para la optimización de las condiciones materiales y no materiales de existencia de la unidad y sus miembros.

Estas familias no construyen estrategias familiares de vida, debido a que las actividades que realizan no están en función, precisamente, de la optimización del bienestar de cada miembro y de todos en general. No pueden salir del círculo cerrado en que se encuentran, pues no poseen volúmenes de capital, la posición en las relaciones de fuerzas entre las clases no es ventajosa, y el capital cultural no ayuda a la elaboración de las estrategias familiares de vida.

La violencia visible e invisible forma parte intrínseca a la vida de estas personas, en lo cual incide el contexto de pobreza, la inestabilidad económica, el bajo nivel cultural y la falta de calificación para alcanzar un empleo decoroso. La conformidad con que asumen la vida, la pasividad y la falta de recursos para buscar alternativas ante la realidad, desencadena paulatinamente formas de violencia, primero sutiles y veladas y luego manifiestas en la cotidianidad.

En estas personas prevalecen los elementos de la cultura de la pobreza, esencialmente el de la dependencia y la marginalidad. La población espera, pasivamente, a que el gobierno o alguna de sus instituciones solucionen sus problemas. No agencian 
empleos o recursos mínimos para cambiar la realidad existente, sino que conviven conformes con lo que poseen $\mathrm{y}$, posteriormente, poco a poco transitan por un estado de violencia creciente.

La violencia se presenta de diferentes formas en estos ciudadanos, tanto en los protagonistas de las historias como en miembros de sus familias. Hay violencia de género con las mujeres como víctimas de los hombres que se han implicado en sus contextos de vida, de esposos, hermanos e hijos que abandonan a sus madres o sea, hay la presencia tanto de la violencia física como la psicológica. La violencia en estas historias es un problema complejo, multideterminado por diversos factores como el contexto de pobreza y la cultura patriarcal, pero no se justifica como acto de descontrol o vía para la resolución de los conflictos en que está inmersa la existencia de estas personas.

\section{Conclusiones}

Los procesos de reproducción social crean las bases para la construcción de estrategias en correspondencia con el habitus adquirido en la formación de los agentes sociales. Las estrategias familiares de vida parten, en la mayoría de los casos, de los conocimientos conscientes o no, legitimados de una generación a otra por la familia.

Las historias de vida realizadas permiten afirmar que las acciones diseñadas no constituyen proyectos de vida con objetivos a largo plazo y se asocian a las actividades asignadas tradicionalmente a cada género. La violencia tanto en los espacios privados como públicos es una consecuencia del estado de vulnerabilidad social en que se encuentran estas personas quienes viven sin planificar más allá de la sobrevivencia diaria y se convierte en un círculo vicioso, lo cual no les permite romper la cultura de la pobreza para entrar en la cultura de la transformación.

\section{Bibliografía}

BOURDIEU, P. L'esprit de famille: raisons pratiques sur la théorie de l'action. Traducción de M. Neufeld. Editions du Seuil, 1994.
. Estrategias de reproducción y modos de dominación. Colección Pedagógica Universitaria, n.37-38, p.1-21, 2002.

CAPEL, H. La definición de lo urbano. Estudios geográficos. n.138-139, 1975.

DÍAZ, M. Familias cubanas entre cambios y estrategias. Revista Enfoques, n.22, 2005. Disponible en: <http:// cubalamano.net>. Acceso en: agos. 2011.

GUTIÉRREZ, A. Teórico-metodológicas de un análisis relacional para los estudios de la pobreza. Ciencia, Docencia y Tecnología, Concepción del Uruguay n.35, p.15-33, 2007. Número Temático Global 2007 en Pobreza y Desarrollo Humano. Disponible en: http://councilscienceeditors.org.

HINTZE S. Capital social y estrategias de supervivencia: reflexiones sobre el capital social de los pobres. Disponible en: <http://tau.org.ar $>$.

MALLIMACI F.; GIMÉNEZ V. Estrategias de investigación cualitativa. Barcelona: Gedisa, 2006.

MOLINA, M. Estrategias de sobrevivencia e inequidades de género: el caso de Argentina en el contexto latinoamericano. Revista Enfoques n. 5, 2006.

PÉREZ SERRANO, E.; BETANCOURT CHACÓN, I.; RODRÍGUEZ GONZÁLEZ, Y. Estrategias familiares de vida: un estudio con enfoque de género en las comunidades La Yabita y Cerro Verde del municipio de Holguín. Ponencia presentada en el VIII Congreso Iberoamericano de Pensamiento, Holguín, Cuba, 2013.

PLA, M. El rigor en la investigación cualitativa. Revista de Atención Primaria, n. 24, p. 295-300, 1999.

RODRÍGUEZ GÓMEZ, G.; GIL FLORES, J.; GARCÍA JIMÉNEZ, E. Metodología de la investigación cualitativa. La Habana: Félix Varela, 1995.

SALTALAMACCHIA, H. Historias de vida: reflexiones a partir de una experiencia de investigación. Puerto Rico: Ediciones CIJUP, 1992.

SARABIA, B. Historias de vida. Revista Reis. n.29, p.165186, 1985.

TORRADO, S. El enfoque de las estrategias familiares de vida en América Latina: orientaciones teóricometodológicas. 2.ed. Buenos Aires: Centro de Estudios Urbanos y Regionales, 1985.

ZABALA, M. Familia y pobreza en Cuba. La Habana: Acuario, 2010.

Recebido em fevereiro de 2014. Aceito em abril de 2014. 